\title{
Triple antibiotic paste in the treatment of a necrotic primary molar: a case report
}

SADJ November 2018, Vol. 73 No. 10 p624 - p626

S Tredoux', R Arnold², GD Buchanan ${ }^{3}$

\section{Declarations of interest}

None. All authors have contributed significantly and all authors are in agreement with the manuscript. This research did not receive any specific grant from funding agencies in the public, commercial, or not-for-profit sectors.

\section{Approval from institution}

Approval has been granted by RESCOM, School of Dentistry, University of Pretoria.

\section{SUMMARY}

This paper reports a case of an eight-year-old male who presented with a primary molar which had previously undergone a pulpotomy. Subsequently pulpal necrosis, internal resorption and mobility of the tooth occurred, complicating the case. A repeat pulpotomy was performed together with the use of triple-antibiotic paste (TAP). At the 10-month follow-up no clinical signs of disease were found. Complete healing of the furcal radiolucency was demonstrated, with bony infill, no mobility and resolution of the previous widening of the periodontal ligament. It appears that TAP may be a viable treatment modality to enable retention of necrotic primary teeth with a poor prognosis.

\section{Keywords}

Pulpotomy, pulpectomy, triple antibiotic paste.

\section{INTRODUCTION}

In cases of symptomatic pulpal involvement and/or necrotic pulps of primary teeth, pulpectomy and canal obturation with resorbable paste systems is the standard treatment modality. ${ }^{1}$ Pulpectomy is however a very complex procedure with a high risk of iatrogenic complications.

Author affiliations:

1. Sheree Tredoux, BChD (UWC), PDD Endodontics (UWC), Department of Odontology, School of Dentistry,

University of Pretoria.

2. René Arnold, BChD (Pret), PGDipDent Paedodontics (Pret), Private Practice.

3. Glynn Dale Buchanan, BChD (Pret), PDD Endodontics (UWC), MSc Dent (Pret), Department of Odontology, School of Dentistry, University of Pretoria.

Corresponding author: Sheree Tredoux

Department of Odontology, 31 Bophelo Rd, Prinshof Campus, Riviera, Pretoria, 0002, South Africa.

Tel +27 (0)123192214 Email: shereetredoux@gmail.com

\section{ACRONYM}

TAP:

triple antibiotic paste

Required are: rubber dam isolation, canal working length determination, cleaning and shaping of canals, irrigation and obturation of dry canals. ${ }^{1,2}$

Due to the complex nature of pulpectomies, clinicians often instead choose the option of extraction and space maintenance. It is generally accepted, however, that best practice is to attempt to maintain a deciduous molar in the arch for as long as realistically possible..$^{2-4}$ It is in the patient's prime interest that an endeavour is made to salvage the tooth until natural exfoliation.

In recent years, triple antibiotic paste (TAP), developed by Sato et $\mathrm{al}^{5}$, has been shown to be effective when used in regenerative endodontic procedures on permanent teeth, including necrotic pulp cases. ${ }^{6,7}$ The body of evidence regarding the effectiveness of TAP used in the treatment of teeth has proven it to be safe for dental use and has demonstrated that the procedure can be used for pulpotomy and pulpectomy in selected deciduous cases. ${ }^{2,4,8}$

\section{CASE REPORT}

An eight-year-old male with non-contributory medical history presented with a main complaint of a fractured restoration on a lower left second primary molar (75) and pain during mastication. The tooth had previously been treated with a pulpotomy (Figure 1).

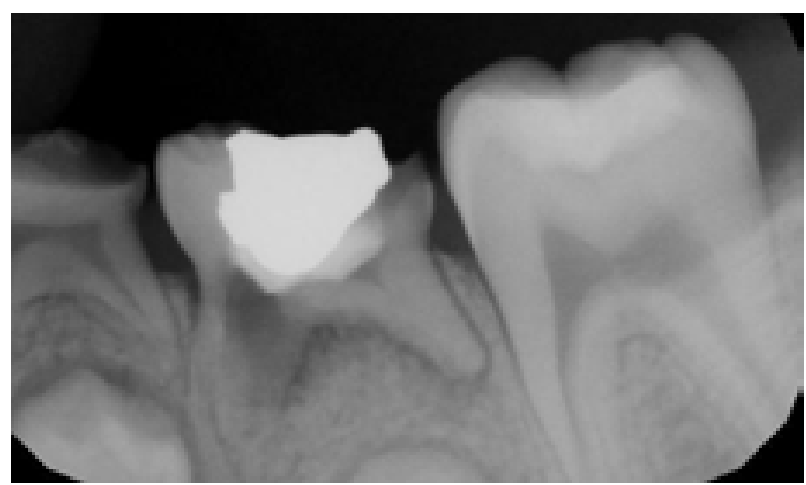

Figure 1. Pre-operative radiograph.

A diagnosis of pulpal necrosis, chronic periapical periodontitis and internal resorption with associated tooth mobility was made, based on the symptoms and radiographic evidence. 
Conventional treatment options including pulpectomy or extraction with placement of a space maintainer were discussed with the caregiver. The decision was made to treat the tooth with a repeat pulpotomy-type procedure and the placement of TAP as a compromise. Consent and assent were obtained.

\section{Treatment performed}

\section{First Appointment}

Following local anaesthesia, the fractured restoration and caries were removed and access to the pulp chamber obtained. A \#10 K-file with RC Prep (Premier, Philadelphia, USA) was used to scout the coronal $5 \mathrm{~mm}$ of the canals. Irrigation was performed with 1\% sodium hypochlorite. TAP was mixed to a ratio of 1:1:1 (using clindamycin, metronidazole and ciprofloxacin) with propylene-glycol as a carrier. The mixture was placed on the pulpal floor and at the canal orifices. A base layer (Kalzinol, Dentsply Detrey, Germany) and final restoration (Permite, SDI, Victoria, Australia) were placed.

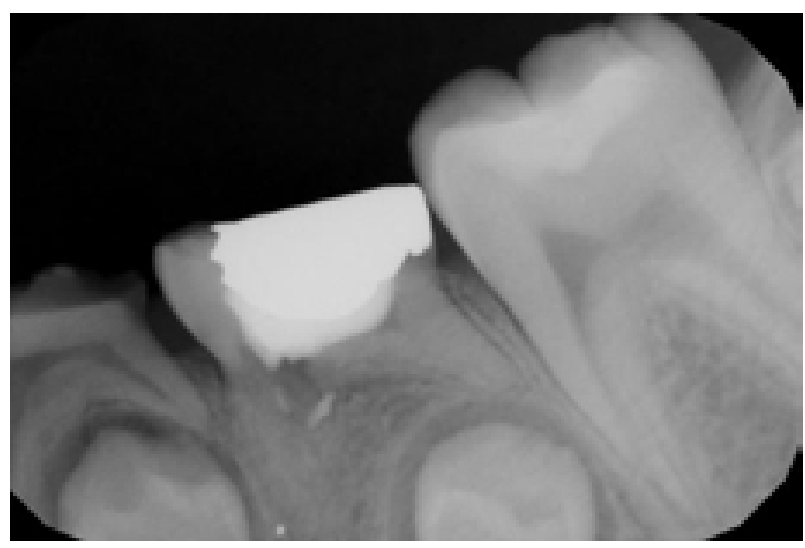

Figure 2. Ten-month follow-up radiograph.

Ten-month follow-up appointment

At the ten-month follow-up, the tooth was asymptomatic, with no signs or symptoms of any active disease clinically or radiographically and the tooth was no longer mobile.

\section{DISCUSSION}

TAP is made by mixing equal parts of metronidazole, minocycline and ciprofloxacin. ${ }^{5}$ Tablets are ground to a powder or capsules are opened and the contents are mixed to a paste with propylene glycol as a carrier. ${ }^{2}$

TAP works by incorporating antimicrobials that are effective against both aerobes and anaerobes. ${ }^{9}$ The most notable and widely recognised use of TAP includes pulp regeneration and revascularisation of immature permanent incisors, in an attempt to stimulate revitalisation and continued root formation. ${ }^{6,7}$ During revascularisation, TAP is used as an interim medicament which is removed at a second appointment.

In contrast to revascularisation procedures, the use of TAP in deciduous teeth involves placement of the paste at the orifice level and/or into the root canals, followed by the placement of a permanent restoration at the same visit. ${ }^{6,8}$ The TAP remains in situ until exfoliation of the deciduous tooth.
Despite its effective antimicrobial action, possible complications and adverse effects of TAP such as tooth discolouration and theoretical antibiotic resistance have been contemplated..$^{7,10}$

Thibodeau suggested replacing the tetracycline antibiotic in TAP with Cefaclor or omitting it totally to avoid possible tooth discolouration. ${ }^{11}$ Additional research is needed to provide clarity on the issue of antibiotic resistance.

TAP has been shown to be effective when used for pulpal treatment in primary teeth. A study comparing pulpectomies to TAP pulpotomies found the 12-month clinical success rate of these procedures to be similar. The clinical success rates of TAP pulpotomies and pulpectomies in this study were $100 \%$ and $96 \%$ respectively at both six and 12-month intervals. ${ }^{2}$

A recent study comparing two different methods of performing TAP pulpotomies showed success with either method, with instrumentation of the coronal pulp being more successful than the non-instrumentation group. ${ }^{12}$

Another study demonstrated the use of TAP without mechanical instrumentation of the canals of necrotic primary teeth to be less effective than traditional pulpectomy.

This study demonstrated that $82.7 \%$ of teeth treated with this procedure presented without clinical symptoms at the two-year mark. ${ }^{8}$ Lower radiographic success rates specifically with non-instrumental TAP pulpotomies after two years (36.7\%) were found. Instrumentation of the coronally accessible pulp tissue therefore appears to be an important factor.

Several authors agree that treatment with TAP should be considered in cases where children are uncooperative and pulpectomy is not possible for clinical reasons or if finances do not allow. ${ }^{2,4,12,13}$

\section{CONCLUSION}

The TAP pulpotomy procedure could allow for retention of a primary molar with a poor prognosis which may otherwise have required extraction and the placement of a space maintainer.

This case illustrates the successful treatment of a necrotic primary molar with a very poor prognosis, using a pulpotomy-type procedure with TAP. While the authors could find no standardised protocol for the TAP pulpotomy, the procedure is simple, not technique sensitive and can easily be adopted in general dental practice. Further research on the subject is required as literature on the use of TAP for primary teeth is sparse.

\section{References}

1. American Academy of Pediatric Dentistry. Guideline on pulp therapy for primary and immature permanent teeth. Pediatr Dent. 2014; 37(6): 212-9.

2. Nakornchai $\mathrm{S}$, Banditsing P, Visetratana N. Clinical evaluation of 3Mix and Vitapex as treatment options for pulpally involved primary molars. Int J Paediatr Dent. 2010; 20(3): 214-21.

3. Aly Ahmed H. Pulpectomy procedures in primary molar teeth. Eur J Gen Dent. 2014; 3(1): 3. 
4. Takushige T, Cruz E V, Asgor Moral A, Hoshino E. Endodontic treatment of primary teeth using a combination of antibacterial drugs. Int Endod J. 2004; 37(2): 132-8.

5. Sato I, Ando-Kurihara N, Kota K, Iwaku M, Hoshino E. Sterilization of infected root-canal dentine by topical application of a mixture of ciprofloxacin, metronidazole and minocycline in situ. Int Endod. J 1996; 29(2): 118-24.

6. Trope M. Regenerative potential of dental pulp. J Endod. 2008; 34(7 SUPPL.): 13-7.

7. Huang GTJ. A paradigm shift in endodontic management of immature teeth: Conservation of stem cells for regeneration. J Dent. 2008; 36(6): 379-86.

8. Trairatvorakul C, Detsomboonrat P. Success rates of a mixture of ciprofloxacin, metronidazole, and minocycline antibiotics used in the non-instrumentation endodontic treatment of mandibular primary molars with carious pulpal involvement. Int J Paediatr Dent. 2012; 22(3): 217-27.
9. Vijayaraghavan R, Mathian V, Sundaram A, Karunakaran R, Vinodh S. Triple antibiotic paste in root canal therapy. J Pharm Bioallied Sci. 2012; 4(6): 230.

10. Slots J. Selection of antimicrobial agents in periodontal therapy. J Periodontal Res. 2002; 37(5): 389-98.

11. Thibodeau B, Trope M. Pulp revascularisation of a necrotic infected immature permanent tooth: case report and review of the literature. Pediatr Dent. 2007; 29(1): 47-50.

12. Pinky C, Shashibhushan K, Vv S. Endodontic treatment of necrosed primary teeth using two different combinations of antibacterial drugs: an in vivo study. J Indian Soc Pedod Prev Dent. 2011; 29(2): 121-7.

13. Prabhakar A, Sridevi E, Raju, Satish V. Endodontic treatment of primary teeth using combination of antibacterial drugs: An in vivo study. J Indian Soc Pedod Prev Dent. 2008; 26(5): 5-10.

\section{Do the CPD questionnaire on page 636}

The Continuous Professional Development (CPD) section provides for twenty general questions and five ethics questions. The section provides members with a valuable source of CPD points whilst also achieving the objective of CPD, to assure continuing education. The importance of continuing professional development should not be underestimated, it is a career-long obligation for practicing professionals.

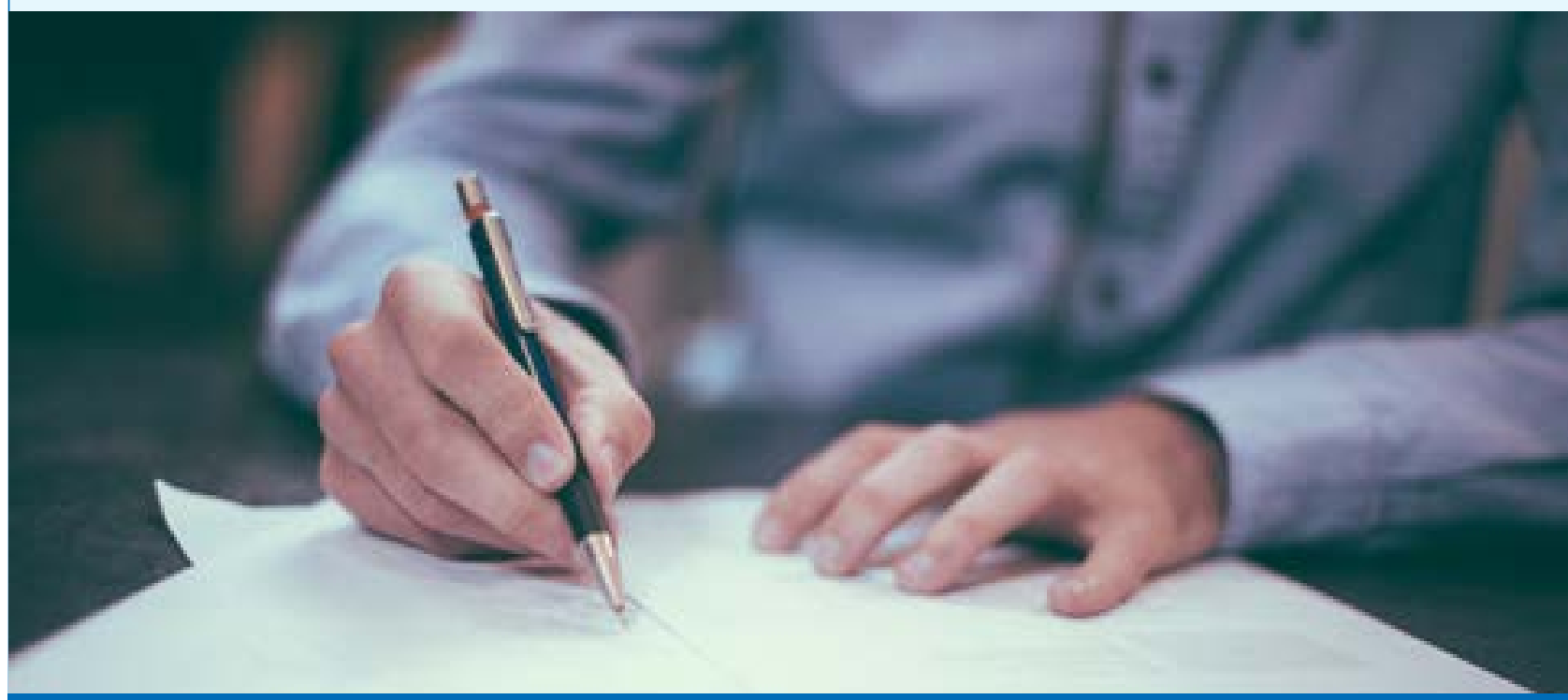

Online CPD in 6 Easy Steps

1 Go to the SADA website www.sada.co.za.

2 Log into the 'member only' section with your unique SADA username and password.

3 Select the CPD navigation tab.

4 Select the questionnaire that you wish to complete.

5 Enter your multiple choice answers. Please note that you have two attempts to obtain at least $70 \%$.

6 View and print your CPD certificate. 